\title{
(6) OPEN ACCESS \\ The association between prehospital care and in-hospital treatment decisions in acute stroke: a cohort study
}

\author{
James P Sheppard, ${ }^{1,2}$ Ruth M Mellor, ${ }^{1}$ Sheila Greenfield, ${ }^{1}$ Jonathan Mant, ${ }^{3}$ \\ Tom Quinn, ${ }^{4}$ David Sandler, ${ }^{5}$ Don Sims, ${ }^{6}$ Satinder Singh, ${ }^{1}$ Matthew Ward, ${ }^{7}$ \\ Richard J McManus, ${ }^{2}$ on behalf of the CLAHRC BBC investigators
}

- Additional material is published online only. To view please visit the journal online (http://dx.doi.org/10.1136/ emermed-2013-203026).

For numbered affiliations see end of article

\section{Correspondence to}

Professor Richard J McManus, Department of Primary Care Health Sciences, NIHR School for Primary Care Research, University of Oxford, Oxford, Oxfordshire OX2 6GG, UK; richard.mcmanus@phc.ox.ac.uk

Received 11 July 2013 Revised 3 September 2013 Accepted 4 September 2013 Published Online First 7 October 2013

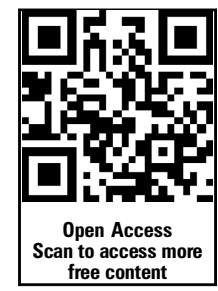

\section{ABSTRACT}

Background Hospital prealerting in acute stroke improves the timeliness of subsequent treatment, but little is known about the impact of prehospital assessments on in-hospital care.

Objective Examine the association between prehospital assessments and notification by emergency medical service staff on the subsequent acute stroke care pathway.

Methods This was a cohort study of linked patient medical records. Consenting patients with a diagnosis of stroke were recruited from two urban hospitals. Data from patient medical records were extracted and entered into a Cox regression analysis to investigate the association between time to $\mathrm{CT}$ request and recording of onset time, stroke recognition (using the Face Arm Speech Test (FAST)) and sending of a prealert message. Results 151 patients (aged $71 \pm 15$ years) travelled to hospital via ambulance and were eligible for this analysis. Time of symptom onset was recorded in 61 (40\%) cases, the FAST test was positive in 114 (75\%) and a prealert message was sent in 65 (44\%). Following adjustment for confounding, patients who had time of onset recorded ( $\mathrm{HR} 0.73,95 \% \mathrm{Cl} 0.52$ to 1.03 ), were FAST-positive (HR $0.54,95 \% \mathrm{Cl} 0.37$ to 0.80 ) or were prealerted ( $\mathrm{HR} 0.26,95 \% \mathrm{Cl} 0.18$ to 0.38$)$, were more likely to receive a timely $\mathrm{CT}$ request in hospital.

Conclusions This study highlights the importance of hospital prealerting, accurate stroke recognition, and recording of onset time. Those not recognised with stroke in a prehospital setting appear to be excluded from the possibility of rapid treatment in hospital, even before they have been seen by a specialist.

\section{INTRODUCTION}

Stroke causes an estimated 5.7 million deaths worldwide and the loss of approximately 50 million disability-adjusted life years (DALYs) every year. ${ }^{1}$ The burden of stroke can be reduced with thrombolytic treatment using alteplase, but the time window for safe and effective treatment is short: thrombolysis is effective at improving functional outcome if administered up to $6 \mathrm{~h}$ following symptom onset, ${ }^{2}$ but only around $4-5 \%$ of stroke patients in developed countries receive this therapy. ${ }^{3}{ }^{4}$ Access to thrombolysis requires timely arrival at hospital, followed by urgent assessment, including brain imaging, to exclude intracranial haemorrhage.
The introduction of the Face Arm Speech Test $(\text { FAST) })^{5}$ and Cincinnati Prehospital Stroke Scale ${ }^{6}$ for use by emergency medical service (EMS) staff has improved prehospital recognition of stroke patients, and completion of the test in suspected stroke patients is a key feature of clinical guidelines and quality standards. ${ }^{7}$ Where a patient is suspected as having stroke, guidelines suggest that a message or 'prealert' is sent to warn hospital staff that a suspected stroke patient is in transit. ${ }^{9}$ This message is relayed to the hospital stroke team which meets the patient in the emergency department and provides specialist care from the moment the patient arrives in hospital.

Hospital prealerting has been shown to improve the timeliness of subsequent treatment in North America and Asia, ${ }^{10}{ }^{11}$ but little is known about its impact in the UK or that of prehospital assessments such as the use of a stroke recognition tool or recording of onset time. The latter is recommended by guidelines in the US, ${ }^{8}$ but receives little attention in the UK. ${ }^{7}{ }^{9}$ Such recording is known to vary widely, ${ }^{3} 12$ and is important because patients with an unknown time of onset are not eligible for prealert or subsequent thrombolysis due to uncertainty about whether they fall within the treatment time window. ${ }^{13}$

The aim of this study was to examine the association between prehospital assessments and prenotification (specifically, recording of symptom onset time, stroke recognition using the FAST test and sending of a hospital prealert) by EMS staff, and subsequent time to CT request and scan and, therefore, eligibility for thrombolysis using routinely collected data from linked hospital and EMS clinical records.

\section{METHODS}

An extended description of the methods used in this study can be found in the online supplementary material.

\section{Study design and setting}

This study used a cohort design. It was conducted as part of a larger project for which the process of recruitment and data collection have been detailed elsewhere. ${ }^{14}$ Briefly, consent to the larger project was sought from patients with a suspected diagnosis of stroke who had been admitted to the acute stroke ward in two urban hospitals (West Midlands, UK) via one ambulance service. A localised EMS protocol for the rapid transfer of suspected stroke patients was in place (see online supplementary 
figure S1). A summary of the patient pathway for acute stroke in the UK is detailed in the online supplementary figure S2.

\section{Selection of participants}

Patients under the care of participating consultant stroke physicians were approached for consent by a member of the research team during their stay on the acute stroke ward during a 9-month period between 1 November 2010 and 31 July 2011. Informed consent was obtained from all patients to permit identifiable patient data to be collected (to allow for data linkage) and only those with capacity (or an available consultee) were approached. Those with a final diagnosis of stroke (defined in their hospital discharge letter) who followed the acute stroke care pathway were included in this analysis.

\section{Data collection}

The records of all consenting patients were scrutinised. Identifiable patient data were used to locate and link hospital and EMS records. Data relating to patient demographics, times to hospital and CT brain scan, prehospital care and route to hospital were extracted from EMS and hospital records. Additional data variables, such as time of nurse triage in the emergency department, time to first assessment by the emergency department consultant, or time to first contact with the stroke team were also sought; however, these data were not routinely recorded in all patient records. Thus, time of CT request and time of CT scan (which were reliably documented by the electronic CT scan booking system) were extracted and used as a 'proxy marker' for timely and effective care.

Missing data were reviewed with source data verification. It was not possible to account for scenarios where assessments were conducted but not documented, or where information about the patients was communicated verbally between healthcare professionals.

\section{Statistical analysis}

All statistical analyses were performed in SPSS V.18.0 (SPSS, Chicago, USA). Descriptive statistics were used to describe the study population, the proportion of patients accessing acute stroke services via ambulance, and the proportion of patients with known onset time and recognised stroke who were prealerted.

For patients arriving at hospital via the EMS, the association between prehospital assessments and time to CT request and scan were examined. Specifically, we used proportional hazards modelling (Cox Regression) to investigate the association between three factors (recording of onset time by the EMS, FAST status (positive or negative/not recorded), or whether a prealert message was sent) and two outcome variables: time from arrival in hospital to CT request (primary outcome), and time from CT request to scan (secondary outcome). These outcome variables were chosen because they are accurately and routinely documented (automatically for every patient), and allow delineation of which part of the in-hospital service is being delayed; the initial decision making of the first attending hospital clinician or the CT scanning department. FAST-negative patients were grouped with those where FAST was not documented for statistical purposes as it was assumed that these patients were similar in not presenting with typical stroke symptoms upon initial assessment.

HRs were adjusted for confounding variables which may have influenced the time to CT request and scan (or the decision to thrombolyse) upon arrival in hospital. In the absence of any established mathematical model describing the scenario examined here, factors were entered into the proportional hazards model as categorical variables using the backwards stepwise method. Full details of included variables, and how they were coded, can be found in online supplementary tables S1 and S2.

The time from ambulance dispatch to key milestones on the stroke pathway was investigated specifically in patients who arrived within $4 \mathrm{~h}$ of symptom onset and, therefore, could be considered for thrombolysis if recognised and not contraindicated. These times were compared in patients where onset time was/was not recorded by EMS staff, the FAST test was completed and positive/negative or a prealert message was/was not sent to the hospital.

Data are presented as means or medians (SD, IQR or $95 \%$ CIs), percentage of the recruited population (unless otherwise stated) and HRs (95\% CI), unadjusted and adjusted.

\section{RESULTS}

\section{Characteristics of the study participants}

A total of 500 patients with stroke were admitted to the acute stroke wards during the recruitment period. Of these, 335 (67\% of those eligible) were approached, and 247 (74\% of those approached) were recruited (figure 1). In all, 210 stroke events (in 208 patients $(84 \%$ of those recruited)) were included in our analysis (39 (16\%) were excluded due to not following the acute stroke care pathway, having a stroke while in hospital, withdrawal, or loss to follow-up). All relevant secondary care records were identified and data extracted (figure 1). A total of 160 patients travelled to hospital via ambulance, of which 151 (94\%) ambulance records were available for data extraction (figure 1). Timings data were available in $>93 \%$ of records for each time point, FAST status in $100 \%$ of records and hospital prealert data in $98 \%$ of located records.

Patients included in the analysis were similar to those not recruited during the study period for all recorded demographics, except that they had lower hospital mortality (table 1). Included patients were elderly (mean age $71 \pm 15$ years) and the majority were men $(54 \%)$ and of white ethnic origin (82\%) (table 1). A total of 21 recruited patients $(10 \%)$ received thrombolysis, and the median time spent in hospital was 7 days (IQR, 4-16 days).

\section{Main results}

Of the 151 patients included in this analysis who travelled to hospital via the EMS, the FAST test was performed in 141 (93\%), and was positive in 114 (75\%). Onset time was recorded by EMS staff in 61 patients, $67 \%$ of those for whom it was known (90 patients in total). In 14 patients (9\% of total), onset time was recorded by EMS staff but not by hospital staff. EMS staff sent a hospital prealert message in 65 cases (44\%).

Patients were transported to hospital by ambulance within a median of $42 \mathrm{~min}$ (IQR, 33-53 min) from ambulance dispatch, regardless of EMS recording of onset time, FAST status, or the sending of a hospital prealert message (figure 2). Where patients were FAST-positive, onset time was recorded, or a prealert message was sent, a CT scan was requested and completed within $1 \mathrm{~h}$ of arrival in hospital (median times to request and scan were 39 and $57 \mathrm{~min}$, respectively (FAST positive patients); 37 and $50 \mathrm{~min}$ (in patients where onset time was documented); 26 and 39 min (prealerted patients)) (figure 2). However, where patients were FAST-negative, or FAST had not been recorded, onset time was not documented, or where the hospital was not pre-alerted, CT requests and scans were delayed (median delay to request and scan was 120 and $155 \mathrm{~min}$, respectively (in patients who were FAST-negative, or FAST was not documented); 97 and $121 \mathrm{~min}$ (in patients where onset time was not documented); 125 and $185 \mathrm{~min}$ (patients not prealerted)) 


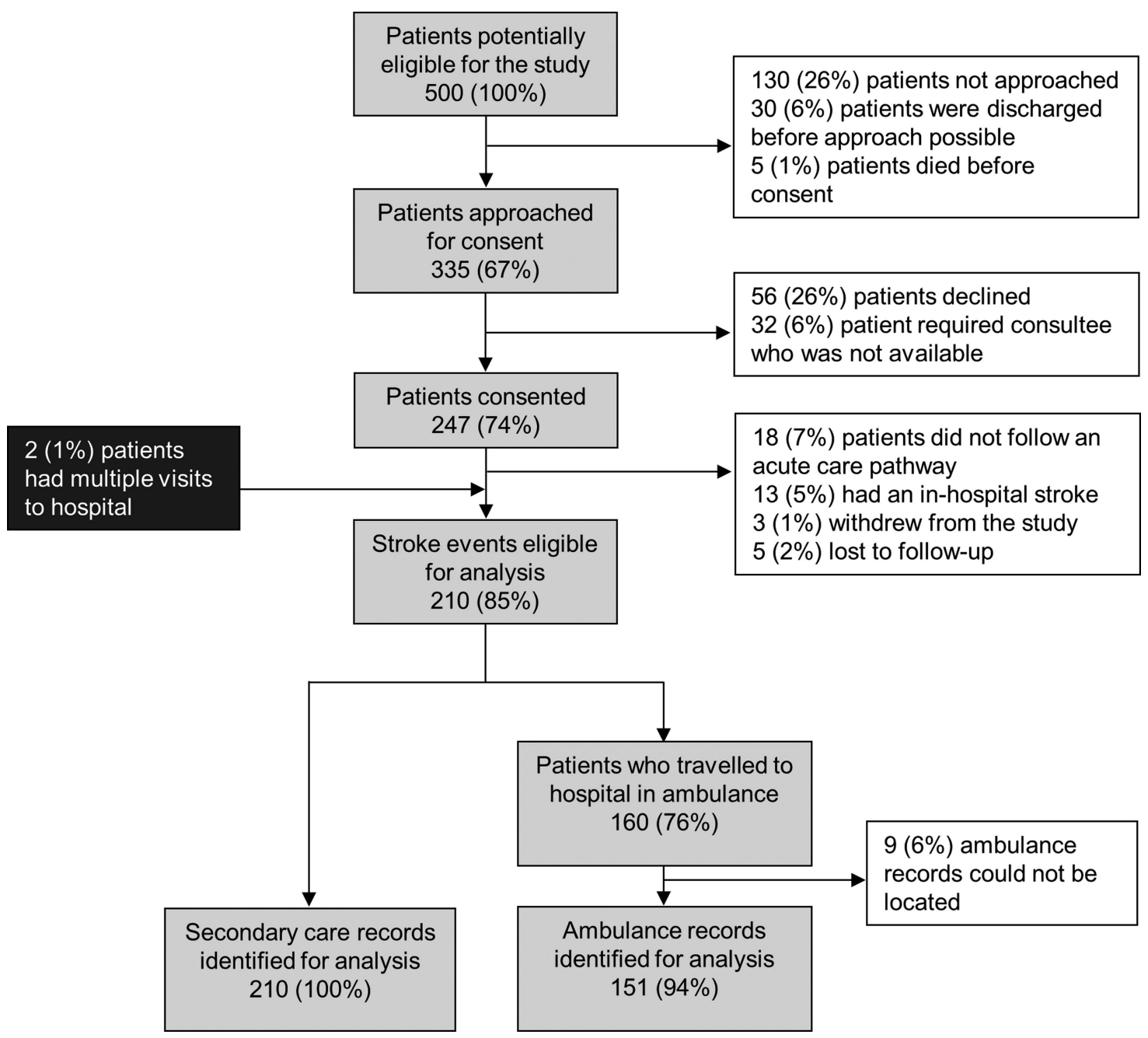

Figure 1 Proportion of patients admitted to hospital with a final diagnosis of stroke included in the final analysis (white boxes indicate excluded patients).

(figure 2). EMS recording of symptom onset time was not associated with delayed CT scan requests in patients who arrived in hospital within $4 \mathrm{~h}$ of symptom onset $(n=73(48 \%))$. However,

Table 1 Characteristics of non-recruited and recruited stroke patients, and those included in the analyses

\begin{tabular}{llll}
\hline Characteristic & $\begin{array}{l}\text { Non-recruited } \\
\text { population }\end{array}$ & $\begin{array}{l}\text { Recruited } \\
\text { population }\end{array}$ & $\begin{array}{l}\text { Included } \\
\text { population* }\end{array}$ \\
\hline $\begin{array}{l}\text { Population (n) } \\
\text { Age (mean } \pm \text { SD) }\end{array}$ & 253 & $\begin{array}{l}247 \\
74 \pm 15 \text { years }\end{array}$ & $\begin{array}{l}71 \pm 15 \text { years } \\
711 \pm 15 \text { years }\end{array}$ \\
$\begin{array}{l}\text { Gender (\% female) } \\
\text { Ethnicity }\end{array}$ & $126(50)$ & $111(45)$ & $95(46)$ \\
$\quad$ White (\%) & $200(79)$ & $202(82)$ & $170(82)$ \\
$\quad$ South Asian (\%) & $32(13)$ & $22(9)$ & $16(8)$ \\
$\quad$ Black (\%) & $7(3)$ & $7(3)$ & $7(3)$ \\
$\quad$ Other (\%) & $4(1)$ & $3(1)$ & $3(1)$ \\
$\quad$ Not stated (\%) & $10(4)$ & $13(5)$ & $12(6)$ \\
$\begin{array}{l}\text { Median time in } \\
\text { hospital (IQR) }\end{array}$ & $9(5.20)$ days & $8(4.17)$ days & $7(4.16)$ days \\
$\begin{array}{l}\text { Died in hospital (\% of } \\
\text { sample population) }\end{array}$ & $51(20)$ & $17(7)$ & $12(6)$ \\
\hline
\end{tabular}

* Recruited population that was included in the analysis (39 recruited patients were excluded because they did not follow acute care pathway, had a stroke while in hospital, withdrew or were lost to follow-up). these patients did experience delays if they were not FAST-positive (median delay $74 \mathrm{~min}$ (to request); $82 \mathrm{~min}$ (to scan)) or not prealerted (median delay $88 \mathrm{~min}$ (to request); 104 min (to scan)), despite being within the time window for thrombolysis treatment upon arrival in hospital.

Kaplan-Meier plots show that patients who had onset time recorded, were FAST-positive, or where the hospital had been prealerted, received consistently faster CT requests within the first $4 \mathrm{~h}$ in hospital (figure 3). Unadjusted HRs suggest the likelihood of receiving a quick CT scan request upon arrival in hospital was increased by $33 \%$ in those with onset time recorded (HR 0.67 , 95\% CI 0.48 to $0.94, \mathrm{p}=0.020), 46 \%$ in FAST-positive patients (HR $0.54,95 \%$ CI 0.37 to $0.80, \mathrm{p}=0.002$ ), and $77 \%$ in patients where the hospital was prealerted prior to arrival (HR 0.23, 95\% CI 0.16 to $0.34, \mathrm{p}<0.001$ ) (table 2). Adjusted analyses gave similar results other than with regard to recording of onset time on time to CT request which was no longer significant (HR 0.73 , $95 \%$ CI 0.52 to $1.03, \mathrm{p}=0.070$ ) (table 2).

\section{DISCUSSION}

Main findings

This study has demonstrated that, in an urban UK setting, prealerted stroke patients are more likely to receive faster assessment upon arrival in hospital. These prealert messages are sent where stroke is recognised, and most frequently where onset time is 

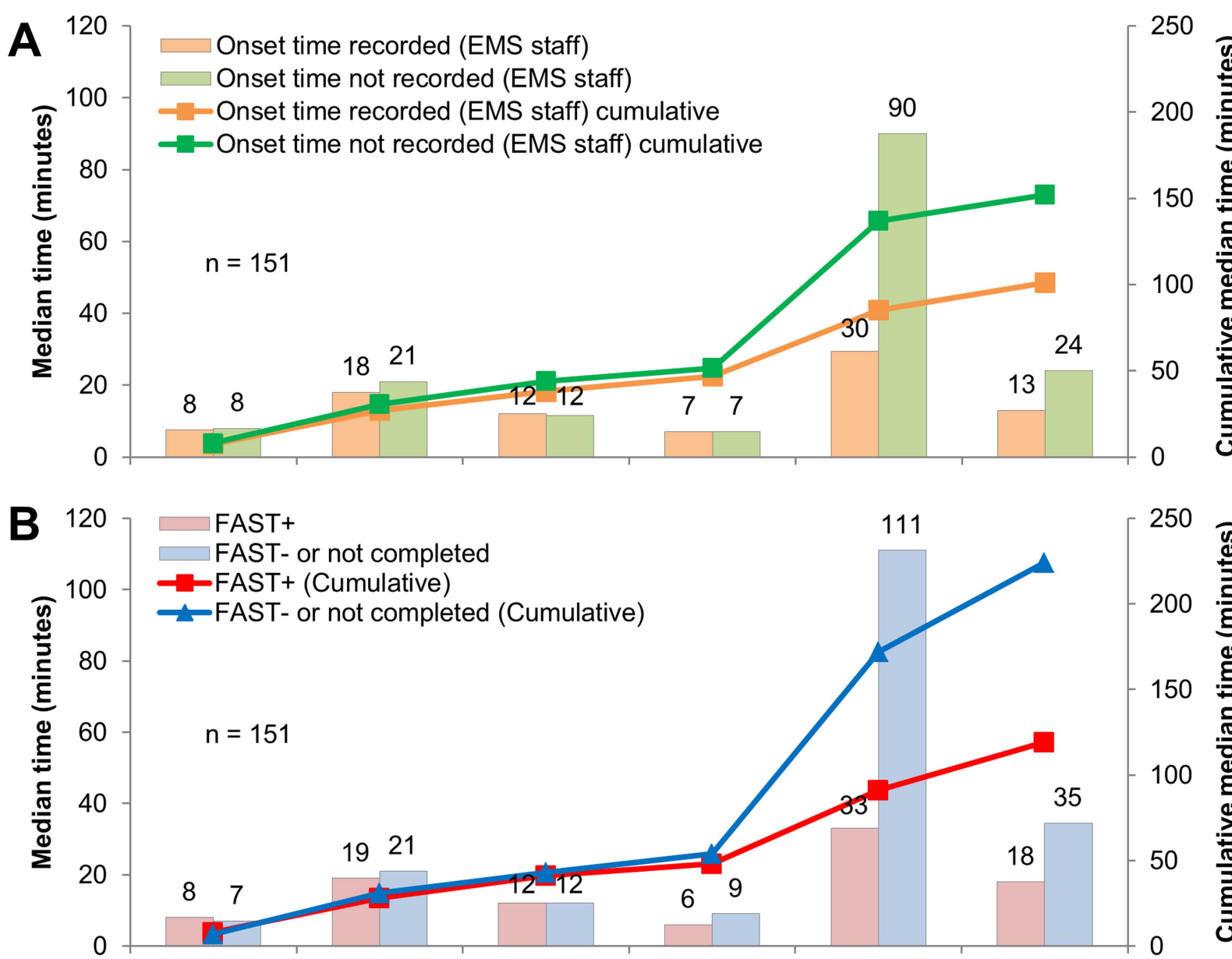

C

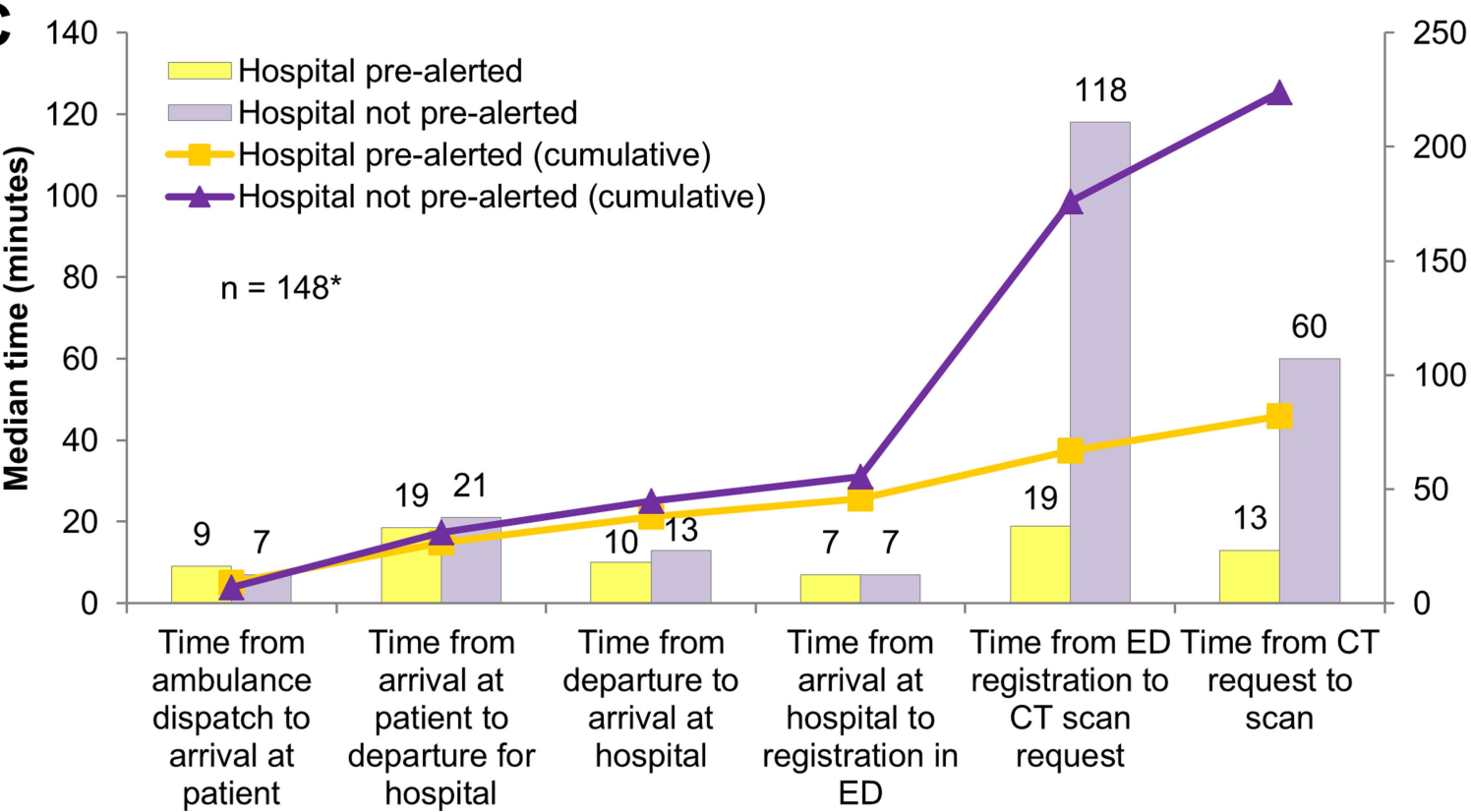

Figure 2 Time to CT scan in patients who travelled to hospital via ambulance by (A) whether or not EMS staff recorded onset time; (B) FAST status; $(C)$ whether or not EMS staff prealerted the hospital. *Prealert data unavailable on three ambulance proformas. EMS, emergency medica service; ED, emergency department; FAST, Face Arm Speech Test.

known. This study highlights the importance of accurate recording of onset time by EMS staff and stroke recognition using an appropriate tool. Our data suggest that some stroke patients are not recognised by current stroke recognition tools, and this has the unintended consequence of delaying stroke patients who might benefit from rapid assessment by a specialist upon arriva in hospital. The use of less specific tools with greater sensitivity should, therefore, be considered to ensure the responsibility of timely stroke recognition falls upon a specialist in hospital rather than EMS staff in a prehospital setting. 

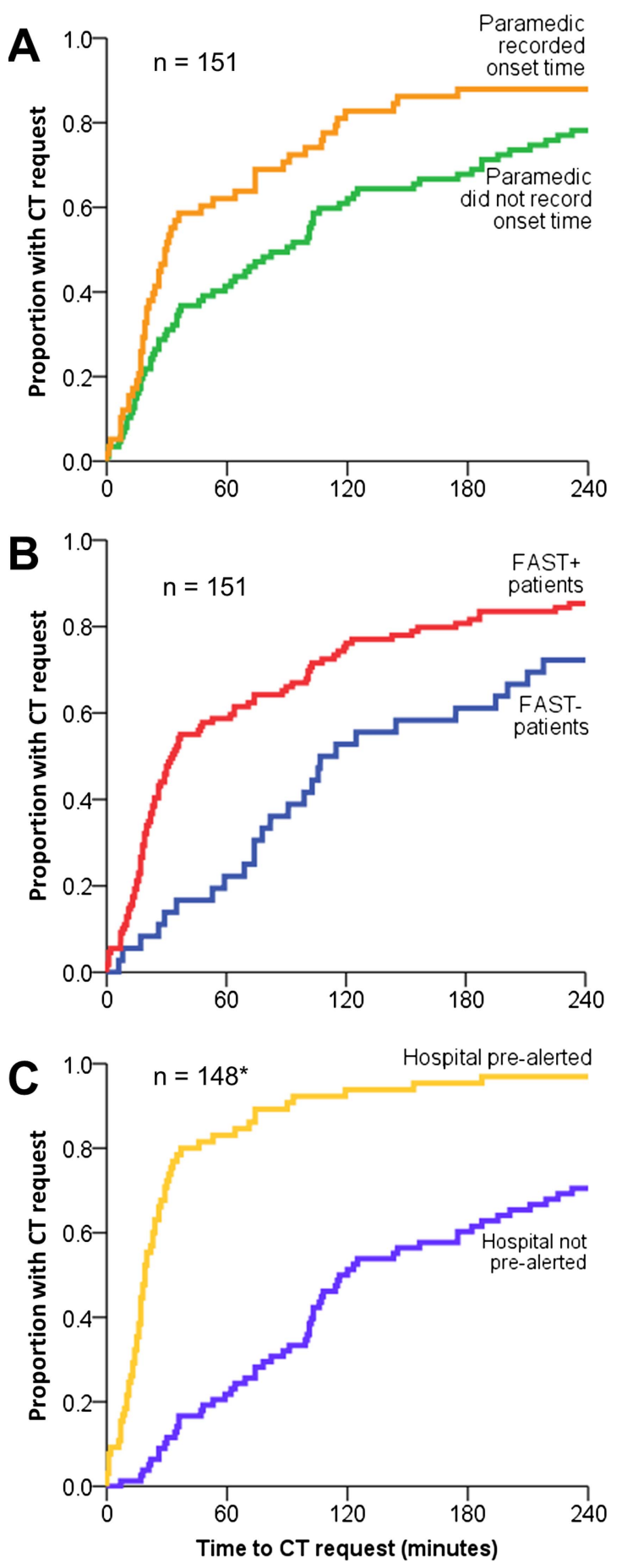

Figure 3 Kaplan-Meier plots showing the proportion of patients with a CT request within the first $4 \mathrm{~h}$ of arrival in hospital for whom: (A) onset time was/was not recorded; (B) the FAST test was positive versus negative/not done; or (C) the hospital was/was not prealerted. *Prealert data unavailable on three ambulance proformas. FAST, Face Arm Speech Test.

\section{Study strengths and limitations}

This was an observational study where patients were recruited prospectively, but data were extracted from medical records after treatment, and so, care must be taken when interpreting the results. Consenting patients with stroke were included which resulted in a population representative of those in the local stroke population other than in terms of early mortality, reflecting the difficulty in gaining consent or assent from very sick patients. ${ }^{15}$ Because only half the potentially eligible patients were recruited, it is possible that our results may have been skewed towards shorter time to CT scan and a higher proportion of thrombolysed patients compared to the total population. Although an attempt was made to sample all patients with stroke, our recruitment was limited by the practicalities of engaging with people presenting $24 \mathrm{~h}$ a day, 7 days a week.

The main rationale for gaining individual consent was to allow data linkage of patient records from different sources which is not otherwise possible in the UK. This meant that data could be collected from $100 \%$ of secondary care records and 94\% of relevant EMS records of consenting participants. Comparatively, only $64 \%$ of ambulance records were identified in a recent national audit of stroke care which does not use such methods. ${ }^{3}$

Only patients with a final diagnosis of stroke were included in this analysis because of difficulties systematically capturing all patients who present with stroke-like symptoms in a prehospital setting. Thus, caution should be taken before drawing conclusions about the relative accuracy of the FAST test or any other stroke recognition tool. The results suggest that up to onequarter of stroke patients were not recognised using the FAST test, but do not show what proportion of stroke mimic patients were correctly triaged as non-strokes on the basis of this test. It was also not possible to determine whether FAST negative patients were suspected as having stroke but ineligible for rapid treatment due to other factors. However, if patients with stroke can be consistently and rapidly transferred to hospital, subsequent management should commence as soon as possible, whether or not thrombolysis is indicated.

\section{Study findings in the context of existing literature}

As with the Emergency Stroke Calls: Obtaining Rapid Telephone Triage (ESCORTT) study ${ }^{16}$ and that of Ramanujam et $a l,{ }^{17}$ in the USA, little difference has been found in the time from ambulance dispatch to arrival in hospital regardless of presumed diagnosis (by ambulance dispatcher and emergency medical staff) with median transit times in both studies of the order of $40 \mathrm{~min}$. However, in keeping with previous work, assessment and communication of that presumed diagnosis does seem to affect subsequent management. Studies from Australia, $^{18} 19$ the USA, ${ }^{10} 20{ }^{21}$ Korea $^{22}$ and Singapore ${ }^{11}$ have all found that prealerting reduces both door-to-imaging and door-to-needle times, although this is the first study to identify these associations in a UK setting. One striking feature of these previous studies is the wide variation in proportion of stroke cases which were prealerted, varying from $22 \%$ to $67 \%,{ }^{11} 18$ with the current study falling somewhere in between at $44 \%$. It is not clear from these studies what the 'correct' proportion of prealerting might be and this merits further study.

The decision to prealert depends on recognition of stroke and identification of potential suitability for thrombolysis. Accurate recording of onset time is required to identify whether patients fall within the appropriate time window for thrombolysis. Previous research suggests that onset time is unknown in between $9 \%$ and $33 \%$ of stroke patients, ${ }^{3} 12$ and a proportion of patients will have wake-up stroke precluding accurate assessment of timing. ${ }^{23}$ In the present study, EMS staff recorded onset time in $67 \%$ of cases where it was known (ie, recorded in either EMS or hospital records). This is lower than audit estimates in the UK which suggest that onset time is recorded in $90 \%$ of cases where it is known in patients with suspected stroke. ${ }^{24}$ These discrepancies may reflect the different populations used 
Table 2 Cox Regression analysis estimating association of prehospital care on times to CT request and scan

\begin{tabular}{|c|c|c|c|c|c|c|c|c|}
\hline \multirow[b]{2}{*}{ Predictor } & \multicolumn{4}{|c|}{ Unadjusted } & \multicolumn{4}{|c|}{ Adjusted* } \\
\hline & HR & \multicolumn{2}{|l|}{$95 \% \mathrm{Cl}$} & $\mathrm{p}$ Value & HR & \multicolumn{2}{|l|}{$95 \% \mathrm{Cl}$} & $\mathrm{p}$ Value \\
\hline \multicolumn{9}{|c|}{ Onset time recorded vs not recorded by EMS } \\
\hline Time to scan request & 0.67 & 0.48 & 0.94 & 0.020 & 0.73 & 0.52 & 1.03 & 0.070 \\
\hline Time to scan & 0.60 & 0.42 & 0.84 & 0.003 & 0.61 & 0.42 & 0.88 & 0.008 \\
\hline \multicolumn{9}{|l|}{ FAST+ vs FAST-/Not done } \\
\hline Time to scan request & 0.54 & 0.37 & 0.80 & 0.002 & 0.54 & 0.37 & 0.80 & 0.002 \\
\hline Time to scan & 0.74 & 0.50 & 1.09 & 0.123 & 0.72 & 0.48 & 1.08 & 0.108 \\
\hline \multicolumn{9}{|c|}{ Hospital prealerted vs not prealerted by EMS } \\
\hline Time to scan request & 0.23 & 0.16 & 0.34 & $<0.001$ & 0.26 & 0.18 & 0.38 & $<0.001$ \\
\hline Time to scan & 0.46 & 0.32 & 0.65 & $<0.001$ & 0.63 & 0.43 & 0.94 & 0.022 \\
\hline
\end{tabular}

to determine these proportions (diagnosis of stroke vs suspected stroke) but suggest that better elucidation of onset time in the prehospital period might improve subsequent management.

In the present study, FAST-positive patients were significantly more likely to receive timely assessment upon arrival in hospital which demonstrates the importance of accurate and timely stroke recognition using an appropriate validated tool. A number of these tools exist and are used in prehospital settings across the world. ${ }^{5} 625$ The majority focus on face, arm/hand and speech symptoms of stroke (with the exception of the Ontario Pre-hospital Stroke Screen ${ }^{25}$ which also includes leg weakness). ${ }^{5} 6$ It is possible that FAST-negative stroke patients in the present study were not detected because they had a more confusing presentation, thus delaying diagnosis and subsequent request for CT scan. For example, patients with posterior circulation stroke often present with dizziness, nausea and vomiting and nystagmus ${ }^{26}$ and, thus, less likely to be detected by the FAST test. ${ }^{27}$ Since these symptoms are common to other conditions, it is possible that a diagnosis of stroke may have been delayed in these patients. While this warrants further investigation, data were not available to investigate this further in the present study as these more specific diagnoses are not routinely documented in medical records in the UK.

Currently, the Improving Stroke Recognition by Ambulance Services (ISRAS) study $^{28}$ is evaluating the feasibility of EMS staff using the Recognition Of Stroke In the Emergency Room (ROSIER) assessment tool (which includes assessment of visual field defects, a common symptom of posterior stroke) and whether this improves the accuracy of stroke recognition. Regardless of the outcomes of this study, the burden of timely stroke recognition cannot rest solely on the EMS: up to onequarter of acute stroke patients travelling to hospital via ambulance are not recognised as FAST-positive, hence, triage at the point of hospital arrival is also important. This is particularly important in areas where patients recognised with suspected stroke are transported directly to regional specialist stroke centres. ${ }^{29}$ Elsewhere, specialist stroke services may not be available and, thus, timely diagnosis of stroke will rely on the performance of the emergency department physician. It is important that all healthcare professionals working in emergency medicine are aware of the different presentations of stroke and the limitations of stroke recognition tools, such as the FAST test. One solution might be that stroke recognition tools with greater sensitivity (perhaps at the expense of specificity) are considered for use in a prehospital setting to ensure that all potential stroke patients arrive at the correct hospital and receive timely assessment by a specialist. Such a change in service provision would be likely to increase the number of stroke mimics being admitted to hospital, thus any new recommendations must first consider the impact on existing stroke services and resources.

\section{CONCLUSIONS}

The study suggests that providing a hospital prealert message is the most influential 'prehospital' factor in facilitating timely assessment for acute stroke patients upon arrival in hospital and confirms in a UK setting the findings of previous work elsewhere. ${ }^{10} 11 \quad{ }^{18-22}$ However, patients are only prealerted where stroke is recognised and symptom onset time is recorded. Given that less than half the stroke patients in this study were prealerted, perhaps the criteria for this should be relaxed. The use of less specific stroke recognition tools with greater sensitivity could be considered in a prehospital setting to avoid certain stroke patients missing out on timely assessment by a specialist in hospital.

\section{Author affiliations}

${ }^{1}$ Primary Care Clinical Sciences, NIHR School for Primary Care Research, University of Birmingham, Birmingham, West Midlands, UK

${ }^{2}$ Department of Primary Care Health Sciences, NIHR School for Primary Care Research, University of Oxford, Oxford, Oxfordshire, UK

${ }^{3}$ Primary Care Unit, University of Cambridge, Cambridge, Cambridgeshire, UK

${ }^{4}$ Faculty of Health and Medical Sciences, University of Surrey, Guildford, Surrey UK

${ }^{5}$ Heart of England NHS Foundation Trust, Birmingham, West Midlands, UK

${ }^{6}$ University Hospitals Birmingham NHS Foundation Trust, Birmingham, West

Midlands, UK

${ }^{7}$ West Midlands Ambulance Service NHS Trust, Regional Ambulance Headquarters, Dudley, West Midlands, UK

Acknowledgements We would like to thank the staff at the participating hospitals for their assistance with recruitment and data collection. We would also like to thank the staff at the participating ambulance Trust for their assistance in locating records. Our thanks to Sheila Bailey and Anita Martin for their administrative support, and to Syed Ahmed for building and maintaining the study database. We would also like to thank Dr David Jenkinson, Roger Holder and Dr Linda Nichols for their statistical support. Finally, we want to thank the consultants and registrars from the emergency department at the University Hospitals

Birmingham NHS Foundation Trust for their views and feedback on our work during this project.

Collaborators Collaborations for Leadership in Applied Health Research and Care for Birmingham and Black Country investigators include: Peter Carr: Heart of England NHS Foundation Trust. Brin Helliwell, Cristina Nand and Norman Phillips: Lay members of Steering Group. Rob Scott: Birmingham and Midland Eye Centre. 
Contributors RJMCM and JPS had the original idea. JPS undertook the analyses and wrote the first draft with RJMcM, TQ and JM. All authors contributed to protocol development, refined the manuscript and approved the final version. RJMcM is the guarantor.

Funding This work was supported by the National Institute for Health Research (NIHR) as part of the Collaborations for Leadership in Applied Health Research and Care (CLAHRC) programme for Birmingham and Black Country. RJMcM holds an NIHR Professorship. The views and opinions expressed are those of the authors and do not necessarily reflect those of the National Health Service, NIHR, or the Department of Health.

Competing interests None.

Ethics approval Approval for this project has been obtained from the National Research Ethics Service (NRES) Committee, London, Queen Square (reference; 09/ H0716/71).

Provenance and peer review Not commissioned; externally peer reviewed.

Data sharing statement Proposals for data sharing to the corresponding author at richard.mcmanus@phc.ox.ac.uk. Consent for data sharing from participants was not obtained, but the presented data are anonymised and risk of identification is low.

Open Access This is an Open Access article distributed in accordance with the Creative Commons Attribution Non Commercial (CC BY-NC 3.0) license, which permits others to distribute, remix, adapt, build upon this work non-commercially, and license their derivative works on different terms, provided the original work is properly cited and the use is non-commercial. See: http://creativecommons.org/ licenses/by-nc/3.0/

\section{REFERENCES}

1 Strong K, Mathers C, Bonita R. Preventing stroke: saving lives around the world. Lancet Neurol 2007;6:182-7.

2 Wardlaw JM, Murray V, Berge E, et al. Recombinant tissue plasminogen activator for acute ischaemic stroke: an updated systematic review and meta-analysis. Lancet 2012;379:2364-72.

3 Henssge U, Hoffman A, Kavanagh S, et al. The National Sentinal Stroke Audit 2010. Round 2011;7:1-74.

4 Nasr DM, Brinjikji W, Cloft HJ, et al. Utilization of intravenous thrombolysis is increasing in the United States. Int I Stroke 2012. 10.1111/j.17474949.2012.00844.x. Epub ahead of print

5 Harbison J, Hossain O, Jenkinson D, et al. Diagnostic accuracy of stroke referrals from primary care, emergency room physicians, and ambulance staff using the face arm speech test. Stroke 2003;34:71-6.

6 Kothari RU, Pancioli A, Liu T, et al. Cincinnati Prehospital Stroke Scale: reproducibility and validity. Ann Emerg Med 1999;33:373-8.

7 National Institute for Health and Care Excellence (NICE). Diagnosis and intial management of acute stroke and transient ischemic attack (TIA). NICE CG068, 2008:

8 Adams HP, del Zoppo G, Alberts MJ, et al. Guidelines for the early management of adults with ischemic stroke: a guideline from the American Heart Association/ American Stroke Association Stroke Council, Clinical Cardiology Council, Cardiovascular Radiology and Intervention Council, and the Atherosclerotic Peripheral Vascular Disease and Quality of Care Outcomes in Research Interdisciplinary Working Groups: The American Academy of Neurology affirms the value of this guideline as an educational tool for neurologists. Circulation 2007;115:e478-534.
9 Quinn T, Black S, Clarke T. Joint Royal Colleges Ambulance Liaison Committee Guideline for Stroke/Transient Ischemic Attack (Update). 2009.

10 Patel MD, Rose KM, O'Brien EC, et al. Prehospital notification by emergency medical services reduces delays in stroke evaluation: findings from the North Carolina stroke care collaborative. Stroke 2011;42:2263-8.

11 Lin CB, Peterson ED, Smith EE, et al. Emergency medical service hospital prenotification is associated with improved evaluation and treatment of acute ischemic stroke. Circulation. Cardiovascular Quality and Outcomes 2012;5: $514-22$.

12 Iosif C, Papathanasiou M, Staboulis E, et al. Social factors influencing hospital arrival time in acute ischemic stroke patients. Neuroradiology 2011;54:361-7.

13 Wahlgren N, Ahmed N, Davalos A, et al. Thrombolysis with alteplase 3-4.5 h after acute ischaemic stroke (SITS-ISTR): an observational study. Lancet 2008;372:1303-9.

14 Sheppard JP, Mellor RM, Bailey SM, et al. Protocol for an observation and implementation study investigating optimisation of the management of stroke and transient ischaemic attack (TIA). BMJ Open 2012;2:1-7.

15 Rose DZ, Kasner SE. Informed consent: the rate-limiting step in acute stroke trials. Front Neurol 2011;2:65.

16 Jones SP, Carter B, Ford GA, et al. The identification of acute stroke: an analysis of emergency calls. Int I Stroke 2012;8:408-12.

17 Ramanujam P, Castillo E, Patel E, et al. Prehospital transport time intervals for acute stroke patients. J Emerg Med 2009;37:40-5.

18 Mosley I, Nicol M, Donnan G, et al. The impact of ambulance practice on acute stroke care. Stroke 2007;38:2765-70.

19 O'Brien W, Crimmins D, Donaldson W, et al. FASTER (Face, Arm, Speech, Time, Emergency Response): experience of Central Coast Stroke Services implementation of a pre-hospital notification system for expedient management of acute stroke. J Clin Neurosci 2012;19:241-5.

20 Abdullah AR, Smith EE, Biddinger PD, et al. Advance hospital notification by EMS in acute stroke is associated with shorter door-to-computed tomography time and increased likelihood of administration of tissue-plasminogen activator. Prehosp Emerg Care 2008;12:426-31.

21 McKinney JS, Mylavarapu K, Lane J, et al. Hospital prenotification of stroke patients by emergency medical senvices improves stroke time targets. I Stroke Cerebrovasc Dis 2011.

22 Kim SK, Lee SY, Bae HJ, et al. Pre-hospital notification reduced the door-to-needle time for iv t-PA in acute ischaemic stroke. Eur I Neurol 2009;16:1331-5.

23 Fink JN, Kumar S, Horkan C, et al. The stroke patient who woke up-Clinical and radiological features, including diffusion and perfusion MRI. Stroke 2002;33: 988-93.

24 Shaw D, Siriwarddena AN. Results of Stroke Clinical Performance Indicator Audit. 2012, Cycle 8.

25 Chenkin J, Gladstone DJ, Verbeek PR, et al. Predictive value of the Ontario prehospital stroke screening tool for the identification of patients with acute stroke. Prehosp Emerg Care 2009;13:153-9.

26 Searls DE, Pazdera L, Korbel E, et al. Symptoms and signs of posterior circulation ischemia in the New England medical center posterior circulation registry. Arch Neurol 2012;69:346-51.

27 Gulli G, Markus HS. The use of FAST and ABCD2 scores in posterior circulation, compared with anterior circulation, stroke and transient ischemic attack. J Neurol Neurosurg Psychiatry 2012;83:228-9.

28 Donohoe R, Koutsokeras A. Improving Stroke Recognition by Ambulance Services (ISRAS). UK Clinical Research Network: Portfolio Database, 2012.

29 Song S, Saver J. Growth of regional acute stroke systems of care in the United States in the first decade of the 21st century. Stroke 2012;43: 1975-8. 